\title{
Enhancing by Saliency-guided Decolorization
}

\author{
Codruta Orniana Ancuti, Cosmin Ancuti and Phillipe Bekaert \\ Hasselt University - tUL -IBBT, Expertise Center for Digital Media \\ Wetenschapspark 2, Diepenbeek, 3590, Belgium
}

\begin{abstract}
This paper introduces an effective decolorization algorithm that preserves the appearance of the original color image. Guided by the original saliency, the method blends the luminance and the chrominance information in order to conserve the initial color disparity while enhancing the chromatic contrast. As a result, our straightforward fusing strategy generates a new spatial distribution that discriminates better the illuminated areas and color features. Since we do not employ quantization or a per-pixel optimization (computationally expensive), the algorithm has a linear runtime, and depending on the image resolution it could be used in real-time applications. Extensive experiments and a comprehensive evaluation against existing state-of-the-art methods demonstrate the potential of our grayscale operator. Furthermore, since the method accurately preserves the finest details while enhancing the chromatic contrast, the utility and versatility of our operator have been proved for several other challenging applications such as video decolorization, detail enhancement, single image dehazing and segmentation under different illuminants.
\end{abstract}

\section{Introduction}

Image decolorization is important in several applications (e.g. monochrome printing, medical imaging, monochrome image processing, stylization). Standard conversion, found in commercial image editing software, neglects the color distribution, and as a result it is commonly unable to conserve the discriminability of the original chromatic contrast (see figure 1). Mapping three dimensional color information onto a single dimension while still preserving the original appearance, contrast and finest details is not a trivial task.

In the last years several technique have been introduced in the literature. Roughly, the decolorization techniques can be grouped in local [10, 19, 3, 22] and global [11,14] approaches. Among the techniques of the first class, Gooch et al. [10] introduced an optimization technique that iteratively searches the gray levels that best represent the color differences between all color pairs. Similarly, the method of Rasche et al. [19] seeks to optimize a quadratic objective function that incorporates both contrast preservation and luminance consistency. Smith et al. [22] developed a two-step algorithm that employs an unsharp mask-related strategy to emphasize the finest transitions. On the other hand, the global strategy of Grundland and Dodgson [11] performs a dimensionality reduction using the predominant component analysis. This approach does not take into consideration chromatic differences that are spatially distant, mapping in some cases different colors into very similar grayscale levels. Recently, Kim et al. [14] have optimized the Gooch et al. [10] method via nonlinear global mapping. Even more computationally effective, this strategy did not solve the problems of the Gooch et al. [10] approach risking to blur some of the fine details. In general, due to quantization strategies or prohibitive function optimization, the existing approaches fail to render the original image look and to preserve the finest details and the luminance consistency (shadows and highlights should not be reversed). Additionally, most of the existing approaches are computationally expensive.

Different than existing methods, we argue that the concept of image decolorization is not to generate a perfect optical match, but rather to obtain a plausible image that maintains the overall appearance and primary the contrast of the most salient regions. Our straightforward operator performs a global chromatic mapping that acts similarly as color filters [1]. In our scheme, the luminance level is progressively augmented by the chromatic variation of the salient information. Generally considered an important feature, saliency was not addressed directly in previous approaches. After the monochromatic luminance channel is filtered and stored as a reference, the luminance values are computed pixelwise by mixing both saturation and hue values, creating a new spatial distribution with an increased contrast of the interest regions. All the precomputed values are normalized in order to fit the entire intensity range. The intensity is re-balanced in order to conserve the amount of glare in the initial image. For extreme lighting conditions, we apply several constraints in order to avoid clipping and fading of 


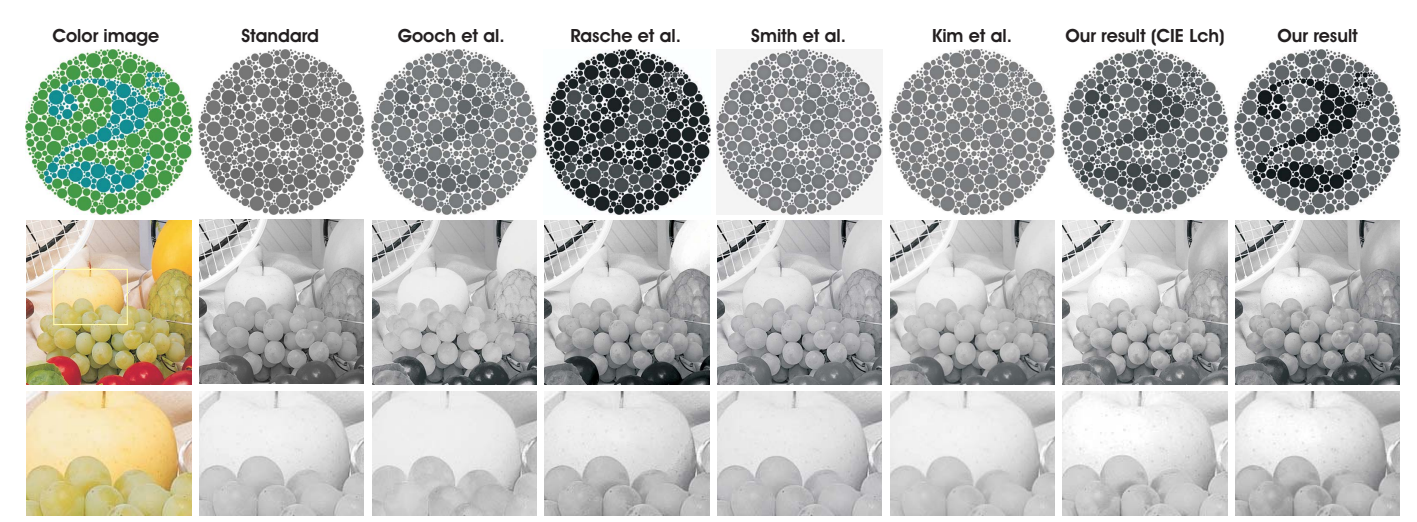

Figure 1. Grayscale transformations. From left to right: initial color image, standard conversion, Gooch et al. [2005], Rasche et al. [2005], Smith et al. [2008], Kim et al. [2009], our method in $C I E L^{*} c^{*} h^{*}$ and our method in $H S L$ color space $(\kappa=2, \gamma=0.7, \chi=0.9$, $\eta=0.2, \phi=200^{\circ}$ ). Notice the details of the highlighted areas of the apple and grapes. The reader is referred to the supplementary material for the complete set of results.

the apparent details.

Our method combines the advantages of the global and local operators being less vulnerable to artifacts due to the continuous global color mapping (free of artifacts) while still preserving the local contrast and details due to the saliency-based strategy and several employed constraints. Furthermore, our strategy is computationally effective being as well validated perceptually by an extensive experiment.

As our decolorization is accurate and preserves finest details, we can exploit variations in chromacity as well as luminance to enhance color images simply, blending the processed luminance levels with the initial color. Observed also by Gooch et al. [10], the human visual system has better spatial acuity in luminance than in chrominance; by fusing the chrominance with co-located luminance changes, weak chroma variations are rendered sharper and better-localized. To the best of our knowledge we are the first to demonstrate such wide applicability of a grayscale operator. We obtain comparable results with specialized techniques for tasks like segmentation under different illuminants, detail enhancement and image dehazing. Furthermore, we show that our algorithm can yield consistent results as well for video sequences.

\section{Our Approach}

As we have previously mentioned, our algorithm aims to increase the contrast of visually salient areas while maintaining the average gray shades that already exist in the image. Our operator is based on a global chromatic mapping that is motivated by psychophysical experiments [6]. Calabria and Fairchild [6] showed that achromatic images are perceived to have a significantly higher level of contrast than images with only $20 \%$ of the original image chroma. The contrast increases monotonically for images with chroma levels above this threshold. Practically, en- hancing the contrast of the luminance based on the chrominance information by a given degree $\gamma$ can be expressed as:

$$
\mathcal{L}=L(1+\gamma S)
$$

where $L$ is the monochromatic normalized value of the luminance channel while the saturation $S$ is defined as the colorfulness of an area judged in proportion with its lightness. This basic expression represents the skeleton of the Helmholtz-Kohlrausch effect [8] and inspired as well some of the previous decolorization techniques [10, 11, 18, 22].

Different than previous strategies, in our approach, to integrate the chromatic polarity gain of the opponent colors (e.g. red pixels need to be mapped lighter when green pixels became darken), the output luminance value $\mathcal{L}$ is computed based on the following chromatic filtering formula:

$$
\mathcal{L}=L(1+\gamma \cos (H \kappa+\phi) S)
$$

where $H$ represents the hue, $L$ is the luminance, $S$ is the saturation while $\gamma, \kappa$ and $\phi$ are some parameters that are discussed in the subsequent paragraphs. Even though our operator shown robustness in preserving the initial color contrast discriminability and details as well in the perceptually uniform $C I E L^{*} c^{*} h^{*}$ [8] color space (with the luminance/lightness $-L^{*}$, hue - $h^{*}$ while the saturation is computed as $c^{*} / L^{*}$ ) (see our results in $C I E L^{*} c^{*} h^{*}$ shown in Figure 1) in this paper we opted for the HSV/HSL color space. In our experiments, besides being faster, HSV/HSL shown to be more accurate and robust while adjusting parameters.

The parameter $\kappa$ represents the period being set to $\kappa=2$ (default value) in order to preserve the color opponency. Briefly, the well-known opponency theory introduced by Ewald Hering (1878) assumes that our vision perception is characterized by three counterbalancing processes: blackwhite, yellow-blue, and red-green. Practically, our decolorized images display complementary gray levels for these 


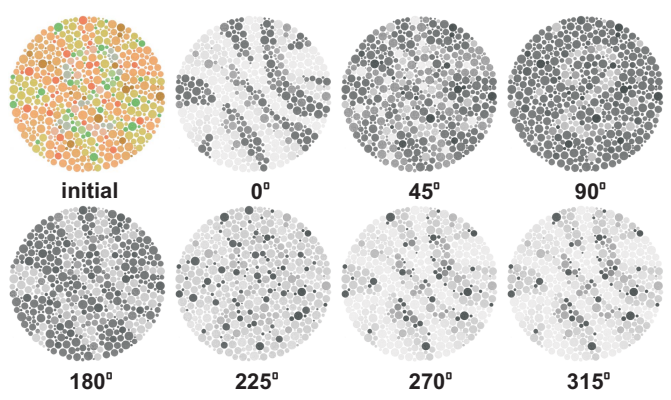

Figure 2. Direct influence of the offset angle $\phi$ to the grayscale representation of the original color image. Note that our approach for entire range of the hue offset angle $\phi$ is able to preserve the initial white color of the background margins. The rest of the parameters are set to their default values.

color pairs. As shown in Figure 4, this default value yields accurate preservation of the most salient regions extracted with the model of Itti et al. [13] that is characterized by a color map built on the Hering's color opponent system. Moreover, for $\kappa=2$ we observed that the range of offset angle $\phi$ can be highly compressed but also tuning the $\gamma$ parameter in a larger range does not introduce significant degradation.

The parameter $\phi$ represents the offset angle of the color wheel $\left(0-360^{\circ}\right)$ (see Figure 2). Its main effect is to set the chromatic enhancing filter on the offset position that best advantages the hue from the region that has lost its saliency due to the decolorization. By adjusting this coefficient, the shades are re-mapped to different gradients. The subsequent subsection goes into detail about how to optimally set this parameter.

The coefficient $\gamma$ tempers the impact of the saturation and acts like a modulator that controls the amount of chromatic contrast. This parameter secures a linear dependency between saturation and global contrast that makes intense saturated images to be rendered with a higher global contrast. To generate the results in this paper was used the default value $\gamma=0.7$ (the only exception is for the dehazing application when $\gamma=2$ ).

In our scheme the multiplication by cosine function plays an important role since it polarizes the $L S$ (luminance-saturation) gain value (equation 2) according with the period $\kappa$. A similar idea was used as well in the previous work of Gooch et al. [10] but using two principal opponent axis. In Grundland and Dodgson [11] the cosine could be approximated by a Gaussian distribution that is more prone to introduce artifacts such as clipping and fading of the details. In the absence of the cosine, if $L$ and $S$ have comparable levels then after the post-normalization process the new value of the luminance remains almost constant. If $L$ and $S$ have different normalized values then the new luminance variation is determined by the saturation.
In some particular cases (e.g. compression and color space non-uniformity) the general equation 2 might yield discontinuities mainly in the neighbor regions of the highlighted areas. In order to solve these issues, for the pixels of the regions with the detected deviations (based on the $\mu$ and $\nu$ thresholds), the $L S$ (luminance-saturation) gain is replaced by the average value of $\overline{\mathcal{L S}}$ :

$$
\overline{\mathcal{L S}}=\left(\sum_{\Omega} L_{x, y} S_{x, y}\right) / N \quad S_{x, y} \geq \mu \text { and } L_{x, y} \geq \nu
$$

where $\mu$ and $\nu$ are thresholds that filter highlighted regions without discontinuities, while $N$ represents the total number of image pixels in the considered region $\Omega$. The values of the thresholds that compensate for the saturation and intensity, $\mu$ and $\nu$, are dependent on the selected color space. In this paper we generate the results using the default values: $\mu=0.1$ and $\nu=0.6$.

Adding previous constraints to equation 2, the luminance $\mathcal{L}_{\text {constr }}$ can now be expressed as:

$\mathcal{L}_{\text {constr }}= \begin{cases}L+\gamma \overline{\mathcal{L} \mathcal{S}} \cos (H \kappa+\phi) & , \quad S \leq \mu \text { and } L \geq \nu \\ L[1+\gamma \cos (H \kappa+\phi) S] \quad, \quad \text { otherwise }\end{cases}$

The next step is to normalize the luminance values to match the initial range:

$$
\mathcal{L}_{\text {res }}=\chi \frac{\mathcal{L}_{\text {constr }}-\mathcal{L}_{\text {min }}}{\mathcal{L}_{\text {max }}-\mathcal{L}_{\text {min }}}
$$

where $\mathcal{L}_{\text {min }}, \mathcal{L}_{\text {max }}$ are the maximum and the minimum values after equation 4 has been computed on the entire image. The parameter $\chi$ (default value, $\chi=0.9$ ) adjusts the global intensity being important to avoid an image over/underexposure look or scene re-illumination.

A general drawback of previous techniques is the loss of the details from clipping or fading that are more visually noticeable on dimmed highlighted and shadowed areas. Qualitatively, although the contrast is overall increased, it is preferable to maintain locally the initial extrema values that practically influence the preservation of the local contrast. We state that for a perceptually uniform output the luminance levels have to remain in a certain range without exceeding the original levels of the input color channels $R, G, B$. Therefore, the intensity is restricted to remain in the local range $[\operatorname{Min}(R, G, B), \operatorname{Max}(R, G, B)]$, securing that the original grayer, whiter or highlighted and darker or shadowed regions of the initial color image are preserved while the contrast is enhanced.

Finally, our decolorized image $\mathcal{I}_{d e c}$ is the result of blending the initial luminance intensity $L$ with the amount of chromatic enhanced luminance $\mathcal{L}_{\text {res }}$ computed with equation 5:

$$
\mathcal{I}_{\text {dec }}=\left(\mathcal{L}_{\text {res }}+\eta L\right) /(\eta+1)
$$


where $\eta$ is the parameter that controls the mixture ratio of the initial $L$ and the enhanced luminance $\mathcal{L}_{\text {res }}$ values. We believe that the way $\eta$ is chosen may create a bridge between the color opponency and the well-known Helmholtz-Kohlrausch effect used in other grayscale conversions [22]. By selecting higher values (in the default range $\eta \in[0,2]$ ) are obtained comparable results as with Helmholtz-Kohlrausch predictors. To decolorize images and for enhancing applications we set this parameter to $\eta=0.2$. For videos, to yield consistent results, an average value of $\eta=1.1$ is more beneficial. By using this value of $\eta$, the transition artifacts between adjacent frames are minimized while the saliency is well preserved.
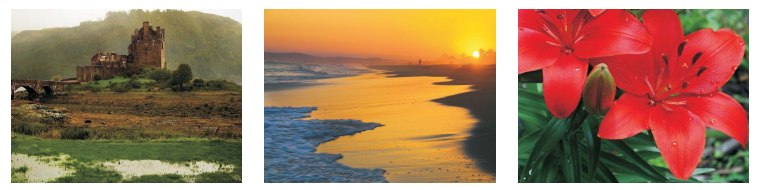

Figure 3. Example of reference images. From left to right the assigned offset angles (HSL color space) are $200^{\circ}, 250^{\circ}$ and $300^{\circ}$.

\subsection{Offset Angle Selection}

In the following we elaborate on how the offset angle parameter $\phi$ is determined. In order to find the optimal offset angle, our algorithm requires a dataset of reference images with an offset angle previously assigned to each image. Based on perceptual preferences by this assignment it is ensured an offset angle value related with the decolorized image version that best conserves the saliency.

The reference images may be real images (see Figure 3 for examples) or synthetically generated using patterns of different color combinations (e.g. red-green, yellow-blue, red-green-blue, red-green-yellow etc.). The allocated offset angle of a reference image is the one associated with the most preferred decolorized version among all possible decolorized variants obtained in the entire range $0^{\circ}-360^{\circ}$. Therefore, in order to simplify the procedure, it is desirable that this range is compressed as much as possible. Theoretically, a various dataset of reference images would ensure a more accurate selection of the offset $\phi$ since, this would better reflect the user's preferences but this would require a great deal of work to create such a large data set. Using this additional information, the offset angle of a given image is determined based on a simple histogram matching operation with a selection of images from the reference image database.

After extensive testing (on +4000 images) we found out that in $H S V / H S L$ color space $(\kappa=2)$ the offset angle mainly needs to cover the $180^{\circ}-360^{\circ}$. Furthermore, we observed that this range can be extremely compressed to the discrete range of $\left[200^{\circ} ; 250^{\circ} ; 300^{\circ}\right]$ with minimal loss of

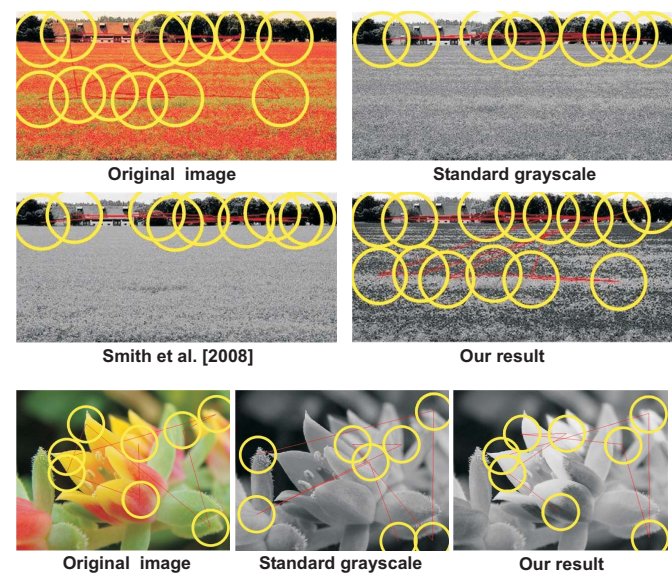

Figure 4. Saliency preservations. Giving a color image, our approach, compared with standard grayscale conversion and Smith et al. [2008], is able to conserve the initial color salient regions (yellow circular regions). Our results have been generated in HSL using default parameters $(\kappa=2, \gamma=0.7, \chi=0.9$, $\eta=0.2$ ) while offset angle was set to $\phi=200^{\circ}$ (top image) and $\phi=250^{\circ}$ (bottom image).

accuracy. This feature can be exploited in real time application but also for video decolorization since it reduces the ambiguity in selecting the offset optimal value of the entire footage.

Instead of considering the entire image in the matching process, for more accurate results, we developed a simple yet effective procedure that selects the optimal offset angle by only taking the most salient regions of the input image into account. This strategy aims to preserve the main salient regions (in both color and decolorized images). In order to identify the salient parts of the image, we have opted for the well-known method of Itti et al. [13] that embraces several biologically plausible steps. The model extracts three different feature maps that are blended into a final saliency map: intensity, orientation and color. The color map of this model is built with the intent of preserving the color opponency. We believe that other more complex models $[15,17]$ that search for the saliency can be applied as well.

Based on this model we first identify the most salient regions in both the color and the standard grayscale (luminance channel) images . For grayscale images we suppressed the color map of the model [13]. A circular region is considered to preserve the saliency only if its position remains relatively constant in both versions (color/grayscale) of the image. We use the default parameters of the model and restrict to a number of maximum five regions (green circles). After comparison, the regions with diminished saliency, seen as regions which lack chromatic contrast, are easily identified. Practically, the distribution of the hue $H$ in these regions determines the selection of the offset angle. (please refer to Figure (1) in the supplementary material for 


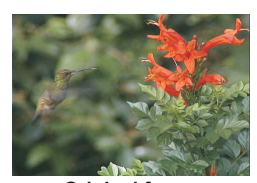

Original frame

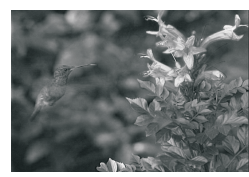

Ours (offset $=300$ )

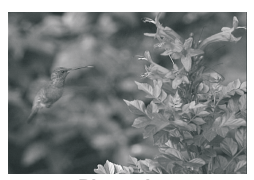

Photoshop

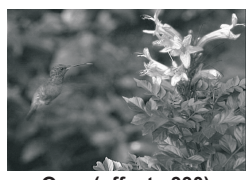

Ours (offset $=330$ )
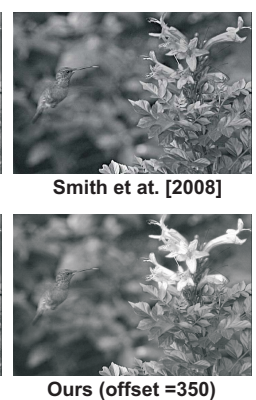

Figure 5. Video decolorization. From left to right: initial color frame, standard grayscale (Photoshop), Smith. et al [2008] and our result for $\phi$ values of $300^{\circ}, 330^{\circ}$ and $350^{\circ}$.

more details about this straightforward procedure).

The results presented in the paper and in the supplementary material, used also in the validation, were generated based on this strategy using the $H S V / H S L$ color space with the default values of the parameters $(\kappa=2, \gamma=0.7$, $\chi=0.9, \eta=0.2)$ and only the discrete range of 3 values $\left(200^{\circ}, 250^{\circ}, 300^{\circ}\right)$ employed for the selected salient regions.

\section{Applications}

Even though designed mainly to effectively decolorize images we found our operator to be particularly well-suited for several other challenging applications.

1. Video Decolorization. Seen as an extension of the image grayscale conversion, decolorizing videos is more complex due to the temporal coherence that needs to be preserved between adjacent frames. Firstly, in our algorithm we search in the entire sequence for the color palette that appears in each image (mostly identified with the static background). For sequences in which the color palette remains relatively constant, a single offset angle $(\phi)$ value computed for the middle frame, yields pleasant results. For more challenging cases (e.g. scenes that alternate very distinctive color palette schemes), the offset $\phi$ is precomputed as an average value of several frames, while constraining its variation in a certain range. To minimize the details loss, for the video decolorization task a value of $\eta=1.1$ (the other parameters are set to their default values) yields consistent outputs. Since this value influences the mean value of the image, the transition artifacts are minimized.

Compared with the approach of Smith et al. [22](see Figure 5) our decolorized frames have a higher discriminative chromatic contrast, thus better preserving the initial saliency. For the entire movie (one of its frames is shown in Figure 5) the optimal offset angle was fount to be $\phi=300^{\circ}$. It should be noted that even though a globally optimal value could be found for the video, there will

always be a trade-off as in some frames different particular details will be lost. As can be observed in Figure 5, by increasing the offset angle value $\left(330^{\circ}, 350^{\circ}\right)$ even though the global contrast is enhanced, on a close inspection some of the details might be lost. For the complete video sequence and some more challenging video examples the reader is referred to the supplementary material.

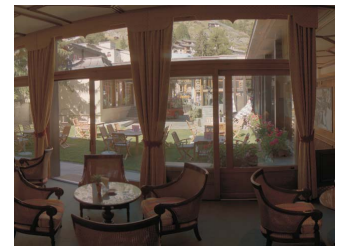

Initial LDR image

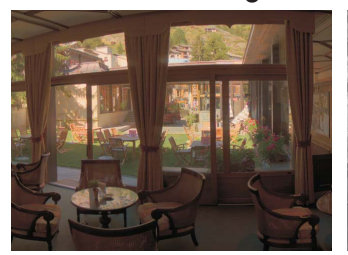

Smith et al. [2006]

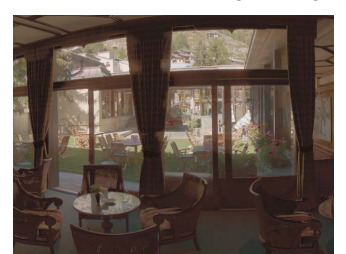

Grundland and Dodgson [2007]

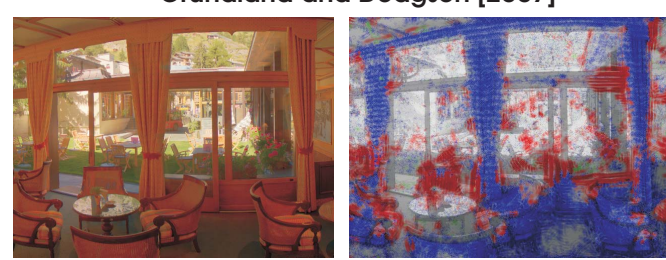

Our result

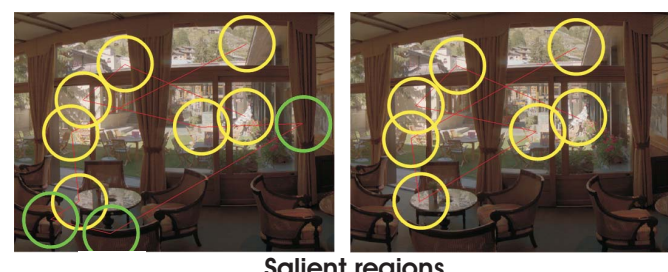

Salient regions

Figure 6. Detail Enhancing. From top to bottom: LDR initial color image and Aydin et al. [2008] quality metric, details enhanced by Smith et al. [2006] , Grundland and Dodgson [2007] and our enhanced results (HSL color space, $\kappa=2, \gamma=0.7$, $\chi=0.9, \eta=0.2$ and $\left.\phi=250^{\circ}\right)$. Notice that our approach is able to enhance outdoor details while interior details (e.g. curtain texture) become more visible. The right column shows the image quality assessment results while the bottom line shows the selected salient regions when the Itti et al. model is employed with color map (left image) and after color map was suppressed (right image). 
2. Image Detail Enhancing. The main goal of image enhancement is to emphasize the image features for display and analysis. As observed by Gooch et al. [10] substituting the luminance channel with the grayscale conversion and recoloring back can yield more pleasant color and grayscale images for printouts. Due to the continuous grayscale mapping and preserving initial details, our technique qualifies for this operation. The LDR image in Figure 6 was obtained by tone mapping with the photoreceptor [20] and therefore the large variations in luminance among regions can not be solved by employing standard operators (e.g. brightness, contrast adjustment available in the commercial tools) that shed details. For this task we employ an iterative colorizing/decolorizing strategy that starts from the offset angle $\phi$, computed as presented in the previous section, and successively blends the resulting luminance after every iteration with the color that has been corrected. For color correction we employed the algorithm of Mantiuk et al. [16] while the rest of the parameters are kept to their default values. As can be seen, our operator has the potential to manipulate global chromatic contrast without using additional information (e.g. images or hardware). The concept is similar to the center-surround [4] frequently used by local enhancing methods, where the mapping varies spatially dependent by the neighborhood of the pixel. However, our strategy applies this concept as a global operation employing the same mapping to all image pixels. Because of the global character of our operator, the presence of haloing artifacts commonly associated with local methods are significantly reduced.

An alternative solution [21] is to manipulate the chrominance for detail restoration. In order to measure the quality of the produced results we opted for the recent metric of Aydin et al. [2] that identifies three classes of contrast changes relative to the original image: loss of visible contrast (green), amplification of invisible contrast (blue) and reversal of visible contrast (red) (practically [2], green is related with blurring while blue and red are related with sharpening operation).

3. Segmentation under Different Illuminants. A known difficulty in image segmentation is that due to the illumination variation highly inconsistent outputs are observed. The recently introduced perceptual color space [7] was designed to solve the illumination invariance. By only minor adjustments, our operator may represent a decent solution for this problem. For this particular task, we disable the constraint of our algorithm that limits the illumination to remain in the range $[\operatorname{Min}(R, G, B), \operatorname{Max}(R, G, B)]$. As presented in the algorithm section parameter $\chi$ controls the global intensity of the images. Therefore, for this task we simply set $\chi$ to the default value (0.9) for the reference image (the one that is relatively well illuminated) while for the second image in order to compensate the differences of illumina-
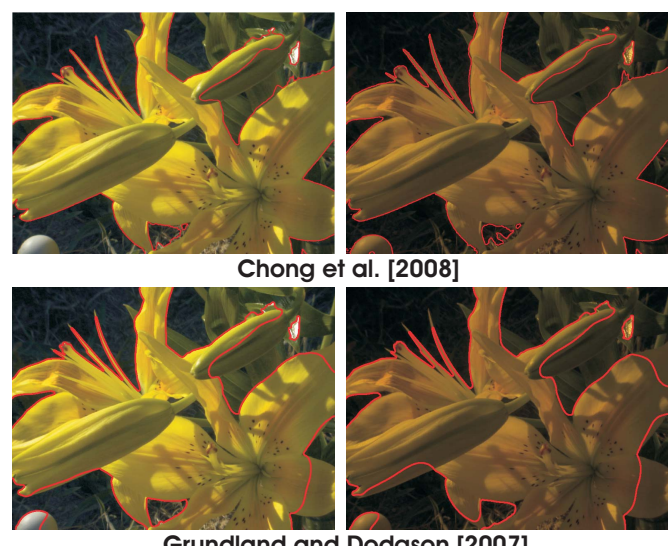

Grundland and Dodgson [2007]
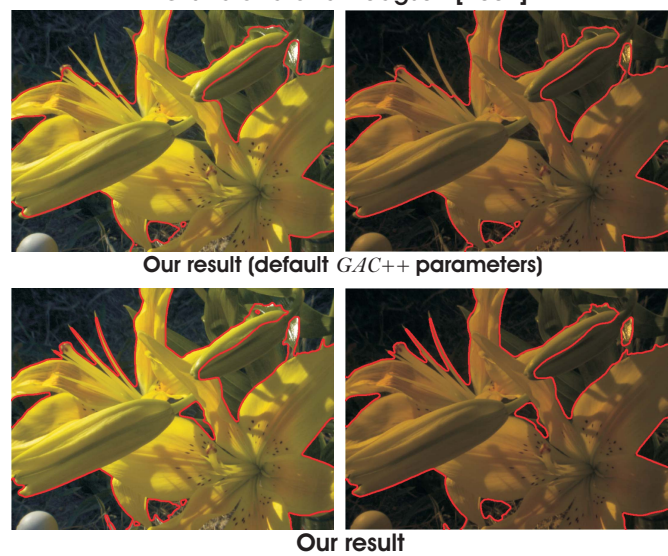

Figure 7. Segmentation under different illuminants. Considering the same image illuminated differently (left-right), the top line displays results obtained by employing the perceptual color space of Chong et al. [2008] after they tweaked parameters for GAC++. Below, the approach of Grundland and Dodgson [2007] yields inconsistent results. The next two lines show our results with and without default GAC++ parameters (we kept the same parameters for our operator). Note that the rounded white object in the left bottom is correctly unselected in our approaches in comparison with the others since the foreground color is yellow. Additionally, the middle opening in the flowers is observed only by our operator when tweaking the GAC++ parameters. To generate our results except for the $\chi$ we used the default parameters in HSL with $\phi=300^{\circ}$.

tion it varies proportionally with the luminance ratio of two corresponding points selected from the foreground.

Since the offset $(\phi)$ selection depends on the salient regions, the scene is reliably decolorized while advantaging objects from the foreground. Running the same algorithm ( the geometric active contour $G A C++{ }^{1}$ ) for the same pair of images used in the work of Chong et al. [7], rendered by varying the light, we obtained comparable results. The same strategy has been applied for the other grayscale operators (Figure 7 displays only the results

\footnotetext{
${ }^{1}$ http://cvsp.cs.ntua.gr/software/GAC++/
} 

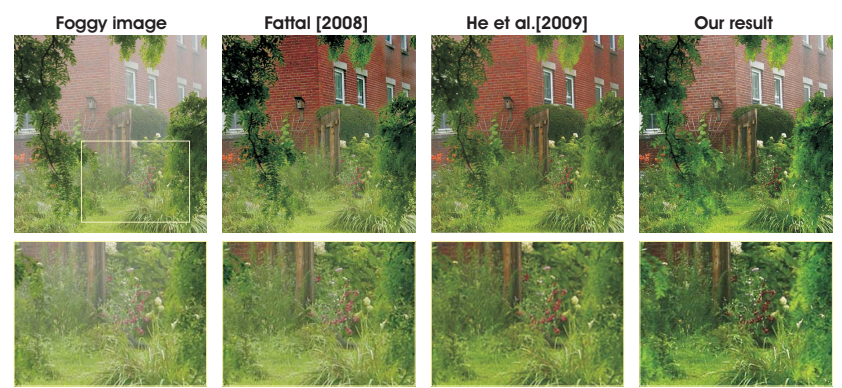

Figure 8. Image dehazing. The initial foggy image, results of two specialized priori-based approaches (Fattal [2008], He et al. [2009]) and our restored output.

of the Decolorize [11] approach). As can be noticed in Figure 7, we firstly employed the $G A C++$ algorithm with default parameters and afterward we applied the $G A C++$ with tweaked parameters for the same grayscale images (the parameters of our operator have been the same as in the first example).

4. Single Image Dehazing. Solutions to this challenging problem have been introduced only recently [9, 12]. In general, the existing strategies succeeded due to strong priors or assumptions that have been made. We also process only a single image and due to the contrast enhancement characteristics of our operator, the yielded results are comparable with the results of such specialized single image dehazing techniques.

For this task it is assumed that due to atmospheric absorption and scattering distant surfaces or objects will appear lighter and less colorful. We used a similar strategy to the one for the detail enhancement application. The algorithm first identifies the hazed areas by computing the luminance difference between initial image and a processed version (with the saturation impact factor is set to $\gamma=2$ ). The global mapping function is constrained by the color distribution of different regions. Therefore, manipulating the contrast difference between the hazed and non-hazed regions the algorithm is able to significantly reduce the degree of haze. Practically, our technique resembles the polarized filters used by photographers to reduce such effects.

\section{Validation of the Grayscale Operator}

Since there is no specialized color-to-gray metric, we managed a perceptually validation similar as the one performed by Cadik [5]. The evaluation considers in addition to our technique and CIEY (luminance channel) several recently introduced decolorization strategies [3, 10, 19, $11,18,22,14]$. For the test 14 volunteers were been involved (9 computer graphics students and researchers and 5 participants with no particular knowledge of computer graphics techniques) in the group of ages (21-45). Be-

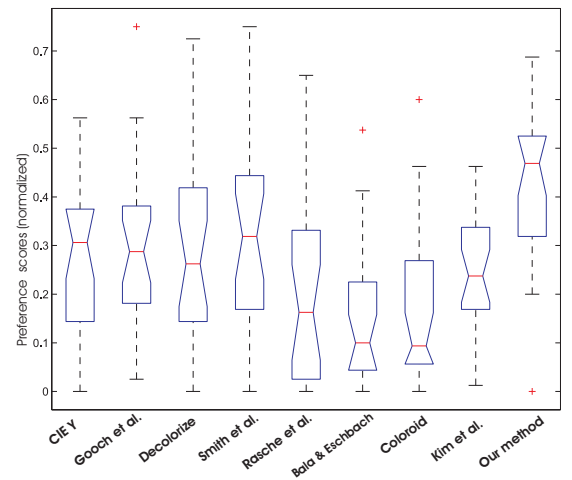

Figure 9. Statistical interpretation of the perceptual evaluation results based on ANOVA ( $p=1.6242 E-06, F=5.67)$.

fore starting the evaluation the grayscale problem has been briefly introduced to the volunteers (written instructions were also available during the test). All volunteers had normal or corrected-to-normal visual acuity and no issues of the color vision. The participants have been asked to rank the grayscale images focusing mainly to the overall appearance, details and contrast preservation. All the grayscale transformations together with the initial color image could be visualized simultaneously by Cooliris $^{2}$, a transparent and very intuitive interface to use.

The images have been visualized on a calibrated 24-inch LCD monitor (Samsung SyncMaster 245B+) at native resolution in a segregated room where the level of darkness was maintained approximately constant for all tests. Different than Cadik's study [5] (where the test took about $20 \mathrm{~min}$ per observer), the volunteers had the entire control resulting in an average time of $90 \mathrm{~min}$ per test. The following ranking strategy has been used: for every set of images the best four results received high scores $(80 \%, 60 \%, 40 \%$ and $20 \%$ ) while the rest of them have been classified as acceptable (scored with 10\%) or not acceptable (not scored). The results have been interpreted statistically using analysis of variance (ANOVA) [23]. As a general remark, analyzing the graphic of Figure 9, the method of Smith et al. [22] and our approach have been ranked as the most perceptually accurate methods. Additionally, as in Cadik's study it can be observed that the methods [19, 10, 14] that optimize an objective function have been classified as less perceptually accurate.

\section{Discussion}

A common problem of existing color to grayscale conversions is the parameters tweaking. Even though seeming complicated on the first glance, the results shown in this paper and supplementary material (e.g. the entire testing set

\footnotetext{
${ }^{2}$ http://www.cooliris.com
} 
of 24 images) have been generated using the default values of the parameters discussed and motivated in section 2 . The reliability of this automatic function of our operator has been obtained due to an extensive analysis based on a large dataset of images. Since psychophysical experiments [6] indicate that (especially) naive observers have large response variability in chroma-contrast experiments it is comprehensible that decolorization methods need to provide a set of intuitive parameters to the users. Besides personalized perception, this alternative fulfills creative interpretations. For example in a natural scene, the shades of gray that portray a water surface can vary over a considerable range. However, for such cases we sought for parameters that are robust against artifacts. The scheme proposed by Smith et al [22] offers several free parameters to the users, but tuning them risks to introduce artifacts close to edges (see supplementary material).

\begin{tabular}{|l|l|l|l|l|l|}
\hline & Gooch & Decolorize & Smith & Kim & Ours \\
\hline Optim. & $4-5 \mathrm{~min}$ & - & $\approx 11 \mathrm{sec}$ & $\approx 0.5 \mathrm{sec}$ & - \\
\hline Unoptim. & - & $\approx 4 \mathrm{sec}$ & - & - & $\approx 1 \mathrm{sec}$ \\
\hline
\end{tabular}

Table 1. Computation times (yielded by authors optimized and unoptimized codes) obtained for a $800 \times 600$ image.

The runtime of our operator is linearly dependent on the image resolution and therefore it is computationally effective being suitable for real-time applications (our Matlab code decolorizes an $640 \times 480$ image in approximatelly 1 second). This is an important improvement compared to the most of the previous approaches (please refer to the table 1).

A limitation of our scheme is the amplification of already existing artifacts in the images or video frames due to compression. Furthermore, manually adjusting the offset angle parameter can yield reversed chromatic contrast (e.g. red may appear darker than green). Our saliency-based strategy may fail in cases when the employed regions (marked with green circle) do not reflect the true salient information of the image. This mainly occurs when specular reflections are identified as salient regions, but also when the object/region of interest is not clearly distinct from the background.

\section{Conclusions}

We presented a straightforward yet effective decolorization algorithm that enhances the contrast of the images while maintaining the initial appearance and quality. Aiming to preserve the original saliency, the monochromatic luminance channel is intensified by mixing both saturation and hue channels. This yields a new spatial distribution, which then is re-balanced in order to conserve the amount of glare impression that characterizes the original version. Besides effective decolorization our operator shown to be appropriate as well for several applications. We currently investigate our operator for scene relighting.

Acknowledgments: The authors would like to thank the reviewers for the suggestions and Cedric Vanaken, Chris Hermans, Mark Gerrits and user-study volunteers for their support.

\section{References}

[1] A. Adams. The negative. Little, Brown and Company, 1981. 1

[2] T. O. Aydin, R. Mantiuk, K. Myszkowski, and H.-S. Seidel. Dynamic range independent image quality assessment. In SIGGRAPH, 2008. 6

[3] R. Bala and R. Eschbach. Spatial color-to-grayscale transform preserving chrominance edge information. In Color Img., 2004. 1, 7

[4] R. Benavente, R. Baldrich, M. Vanrell, and A. Salvatella. Color image enhancement based on perceptual sharpening. In IEEE Int. Conf. Image. Proc. (ICIP), 2003. 6

[5] M. Cadik. Perceptual evaluation of color-to-grayscale image conversions. Computer Graphics Forum, 27(7), 2008. 7

[6] A. Calabria and M. Fairchild. Perceived image contrast and observer preference i: The effects of lightness, chroma, and sharpness manipulations on contrast perception. J. of Img. Sc. and Tech., 2003. 2, 8

[7] H. Y. Chong, S. J. Gortler, and T. Zickler. A perception-based color space for illumination-invariant image processing. SIGGRAPH, 27(3), 2008. 6

[8] M. D. Fairchild. Color Appearance Models, 2nd Ed. Wiley-IS\&T, Chichester, 2005. 2

[9] R. Fattal. Single image dehazing. SIGGRAPH, 27(3):1-9, 2008. 7

[10] A. Gooch, S. C. Olsen, J. Tumblin, and B. Gooch. Color2gray: salience-preserving color removal. SIGGRAPH, 2005. 1, 2, 3, 6, 7

[11] M. Grundland and N. A. Dodgson. Decolorize: Fast, contrast enhancing, color to grayscale conversion. Pattern Recognition, 40(11), 2007. 1, 2, 3, 7

[12] K. He, J. Sun, and X. Tang. Single image haze removal using dark channel prior. In IEEE CVPR, 2009. 7

[13] L. Itti, C. Koch, and E. Niebur. A model of saliency-based visual attention for rapid scene analysis. IEEE TPAMI, 1998. 3, 4

[14] Y. Kim, C. Jang, J. Demouth, and S. Lee. Robust color-to-gray via nonlinear global mapping. SIGGRAPH Asia, 2009. 1, 7

[15] T. Liu, J. Sun, N.-N. Zheng, X. Tang, and H.-Y. Shum. Learning to detect a salient object. In IEEE CVPR, 2007. 4

[16] R. Mantiuk, R. Mantiuk, A. Tomaszewska, and W. Heindrich. Color correction for tone mapping. In Comp. Graph. Forum, 2009. 6

[17] L. Marchesotti, C. Cifarelli, and G. Csurka. A framework for visual saliency detection with applications to image thumbnailing. In IEEE ICCV, 2009. 4

[18] L. Neumann, M. Cadik, and A. Nemcsics. An efficient perceptionbased adaptive color to gray transformation. In Proceedings of Computational Aesthetics 2007, pages 73-80, 2007. 2, 7

[19] K. Rasche, R. Geist, and J. Westall. Re-coloring images for gamuts of lower dimension. Eurographics, 2005. 1, 7

[20] E. Reinhard and K. Devlin. Dynamic range reduction inspired by photoreceptor physiology. IEEE Trans. Vis. Comp. Graph., 2005. 6

[21] K. Smith, G. Krawczyk, K. Myszkowski, and H.-S. Seidel. Beyond tone mapping: Enhanced depiction of tone mapped HDR images. In Computer Graphics Forum, 2006. 6

[22] K. Smith, P.-E. Landes, J. Thollot, and K. Myszkowski. Apparent greyscale: A simple and fast conversion to perceptually accurate images and video. Comp. Graph. Forum, 2008. 1, 2, 4, 5, 7, 8

[23] B. G. Tabachnick and L. S. Fidell. Using Multivariate Statistics. Allyn \& Bacon, 5th edition, 2005. 7 